\title{
An integrated testing system for Spacecraft based on DDS adaptive QoS
}

\author{
Lianbing Huang a , Shunliang Pan, Yuan Li, Pan Zhao, and Guoliang Tian \\ Institute of Manned Space System Engineering, Beijing 100094, China \\ a290633917@qq.com
}

Keywords: DDS, adaptive QoS, test system for spacecraft, publish/subscribe

\begin{abstract}
In order to study the information interaction department of the large complex spacecraft integrated test system. First, we need to analyze the information interaction technology of the spacecraft test system. Through analysis, we use data distribution service (DDS) specification to spacecraft. The information interaction technology of integrated test system for spacecraft based on DDS adaptive QoS. The test system based on this method has the characteristics of high real-time, easy integration and high reliability. It solves the performance bottleneck of the original Client/Server based measurement system.
\end{abstract}

\section{Introduction}

With the extensive application of Internet and rapid development of computer technology, the structure of various application systems is presented. The tendency of internet is central oriented, which puts forward higher requirement for the real-time, dynamic and flexible of communication, and requires the distributed system. Each participants use a flexible communication model and interaction mechanism supported by loose coupling characteristics and QoS (Quality of service). In the space of spacecraft testing, telemetry is done on the spacecraft, and after the downlink, the main test processor (MTP) is forwarded to the remote terminal system (RTS), the client software subscribes the required data from the RTS. From the analysis of the principle of communication, TCP uses the acknowledgement mechanism and retransmission mechanism, flow control and congestion control functions to ensure the reliability of data transmission. But the process is complicated, it is bound to affect the transmission rate of the data; And UDP is a connectionless protocol, the main role is to compress the network data stream into a message form, do not guarantee the order of sending data packets nor properly check, so in the process of transmission possible data loss and disorder phenomenon [1]. However, as the design of the spacecraft is increasingly complex, the amount of downlink telemetry data is getting larger, and when it appears large, the performance bottleneck of the client server mode based data subscription is becoming increasingly prominent when the client concurrent visits to RTS. It is easy to appear such network blocking, data disorder, data losing frame and so on.

In view of the above issues, middleware technology based on subscribing and publishing model can solve the real-time and reliability problems of the integrated test system effectively [2]. At present, there are many models based on subscription and publish, including WebSphere MQ, JMS of IBM and so on [3]. In view of the specific environment of spacecraft testing, this paper uses the open source project OpenDDS as a communication middleware.

\section{Analysis of information interaction requirements of test system}

The spacecraft integrated testing system is a large distributed heterogeneous system, as shown in Figure 1. It has the function to receive test data analysis processing, data warehousing, forwarding source information storage, automatic interpretation, automatic testing and visual display. It's structure is complex, so the demand for more specific information interaction technology, are as follows: 


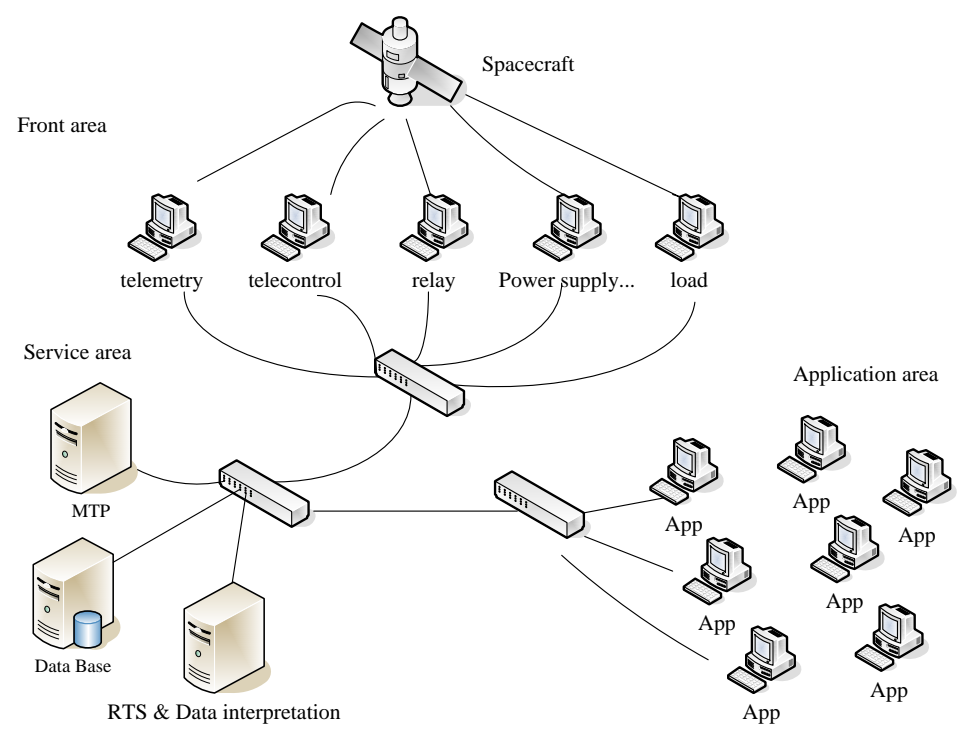

Fig 1 Spacecraft integrated testing system

- Multi data flow information interaction ability. The test data including the whole package source, engineering telemetry, data management telemetry and delayed telemetry, different according to the needs of their own subscription data, which requires the information interactive technology in the integrated test systems can complete;

- Ability to transmit large data. The subsequent spacecraft function is increasingly complex, with the increase of downlink data is fast, especially by the Ethernet downlink data, including their telemetry, image, video and voice information etc. These large data need to be distributed to a integrated test system in a timely and reliable way, and the information interaction capability of the system is very high.

- Ability of information interaction priority. During the test of spacecraft, different subsystems have different data on the degree of urgency and importance, and the real-time requirement for data is different. For example, GNC data has higher priority and real-time requirement. When a specific flight procedure is applied, data interaction should first be preserved. This requires that the test system can be transmitted in an orderly manner according to the priority policy of the information.

- Data sharing capability. At present, after the telemetry data of the integrated test system is parsed by MTP, the RTS is then forwarded two times, which has the risk of losing the sequence of data frames and losing frames. Because of the different source of data, there is also a hidden danger of inconsistent time between database data and data received by different software. This requires that the system has the ability of sharing data of the same source, so as to ensure the consistency of the subscribers' data.

\section{A brief introduction to DDS}

Data Distribution Service (DDS) is an Object Manage Group (OMG), a data centric distributed communication middleware protocol and API standard, is designed to provide unified data distribution mode for system level component integration [4].

\subsection{DDS communication model}

The model mainly includes 5 participants: Data-Object, Publisher, Subscriber, Data Writer and Data Reader, among which the first three are the system owners. To be a participant, data writer is created by publisher; a publisher can create multiple data writers; data readers are created by subscribers, and a subscriber can create multiple data readers. The publisher sends thematic information to the information base; the subscriber sends the subscription theme information to the information base and the information library matches and publics thematic information and subscribe thematic information. Make the relevant QoS policy, and then the publisher sends the corresponding data to the corresponding subscriber. 


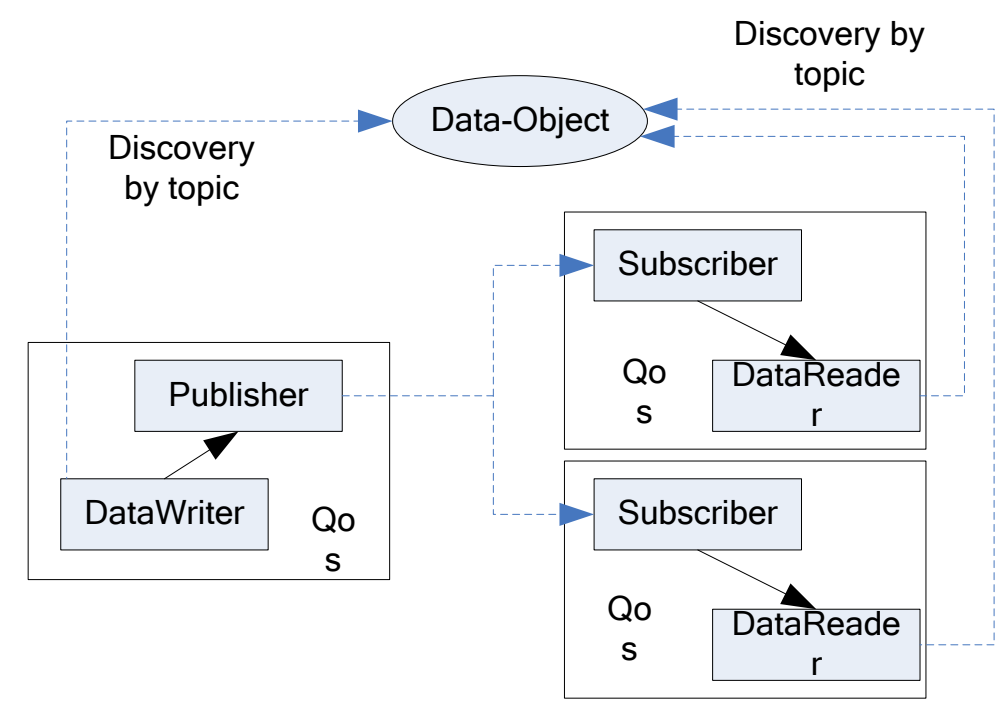

Fig 2 DDS communication model

\subsection{The real mechanism and characteristics of QoS}

The key to the implementation of QoS is to solve the network congestion. There are four strategies to guarantee the implementation of the network [5]:

- Improve the network bandwidth, this method is suitable for LAN, but with the expansion of network scale, it is easy to have the bottleneck problem.

- Retain static resources for application, TDM is to retain static resource technology, usually used in WAN connection, not used in the LAN.

- The shaping technology to ensure the quality, this strategy requires transmission error detection and correction information transmitted in the signal at the same time, so that the user terminal without losing the self-reduction and repair of the original signal. Its disadvantage is that the network overhead is increased and the terminal is highly intelligent.

- Apply the dynamic allocation of resources, intelligent equipment is used to identify the types of data in the network, so as to dynamically allocate the network resources. The method does not need to modify the existing terminal system and application software. This can not only provide a consistent quality of service guarantee throughout the network, but also have good scalability.

At present, open DDS implements its QoS to save static resources for applications and to combine dynamic allocation of resources for application. But from the point of view of application, it finds that it adopts static QoS strategy. After setting up the theme and the writer, it cannot change the QoS setting of the entity. There are some shortcomings, including static settings cannot be completed and network dynamics cannot be applied, do not consider the balance of the whole network and restrict the real-time of information distribution.

\section{Design of integrated test system based on DDS}

\subsection{Information interaction structure of integrated test system}

Based on the analysis of the characteristics of DDS technology, it is known that the use of DDS technology can meet the requirements of the test system for information interaction. This section describes the structure of the integrated testing technology information management structure based on DDS, as shown in the Fig 3. The system structure is divided into 4 levels: the data acquisition layer, the service layer, the DDS information interaction layer and the information control layer. There are different functions between each level. 


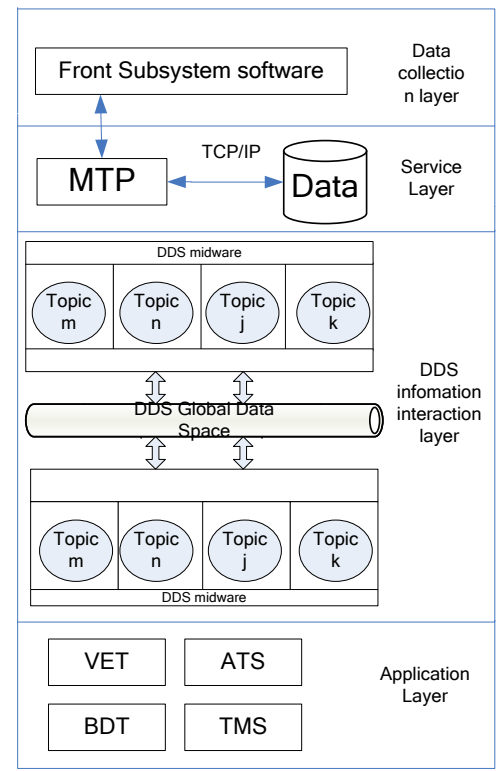

Fig 3 Test system information interaction framework based on DDS

- Data acquisition layer. Responsible for receiving downlink data by spacecraft, send to MTP through TCP/IP.

- Service layer. MTP is responsible for receiving data from the acquisition area and sending data to the database and information warehouse by parsing the data into various themes.

- DDS information interaction layer. The DDS information interaction layer mainly uses the global data space and the information transmission network to integrate each system, and provides a unified information interaction platform for test. The platform manages all the nodes of the testing system, and gets information of each node and sends the information needed by other nodes by issuing and subscribing the theme.

- Application layer. It is mainly composed of automatic interpretation server, visual software and automatic test software. The automatic interpretation server reads the telemetry data and sends the result to the display client; the visual software provides the spacecraft to the monitor. Working status information; automatic test software receives real-time data and auxiliary control decisions of each node; operators analyze them and issue control commands, so as to control the operation of the whole system.

\subsection{Self-adaptive QoS implementation framework}

At present, in the spacecraft testing system, MTP is the only publisher and the rest of the equipment is used as subscribers. One to many adaptive QoS framework is counted, as shown in Fig 4. The framework regulates the QoS requirements of each subscriber according to the network state. To achieve a balanced state, as far as possible to meet all publish subscriptions.

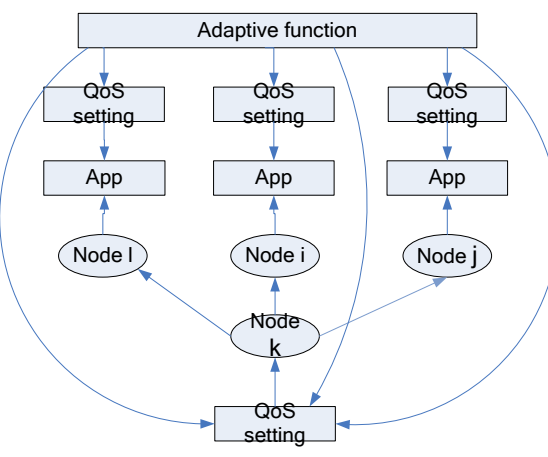

Fig 4 One to many self-adaptive frameworks 
Thereinto, the adaptive function is defined as $\mathrm{Fa}=\mathrm{Fa}(\mathrm{u}, \mathrm{g}, \mathrm{q}, \mathrm{C})$, where $\mathrm{u}$ is the information provided by users, $\mathrm{g}$ is the state information of network resources, $\mathrm{q}$ is the QoS index of data transmission, and $\mathrm{C}$ is the priority of data information transmission. The control object is the allocation of resources for system resources and network resources control. The control decision is to make use of the current network resource status information to determine how the node's QoS changes. Specific control process, shown in Fig 5:

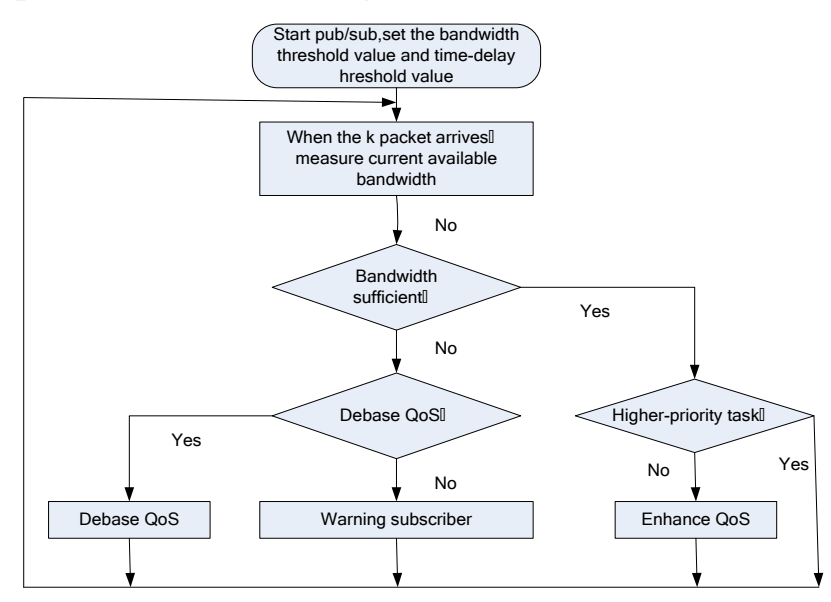

Fig 5 Self-adaptive QoS implementation framework

According to the real-time monitoring of the current network state, and forecast the next state, adjust the QoS in the right time according to the change of the state:

- When the available bandwidth is greater than the set of noisy values, such as the network state is good, there is no remaining bandwidth, keep the original QoS;

- When the available bandwidth is greater than the set of noisy values, such as the network state is good, there is remaining bandwidth, improve the original QoS;

- When the available bandwidth is less than the set values, there may be a network blockage, do not receive a lower QoS node in the current. Reduce the current QoS, notify the user;

\section{Test and analysis}

In this paper, under the test environment of the spacecraft, the experiment of the simulated publication of the test data is carried out. A computer acts as a central node, runs a data warehouse, and a computer acts as a publisher to publish the data, and the rest of the computer as a subscriber. With the same computer configuration, it sends 4 bytes to 4096 bytes at the speed of the 10ms package for 3.6GHz, 8GB RAM, and Windows7. The data delay of the size in different data packets is counted by bytes, and the result of the test shows that the delay of the system is lower than $6 \mathrm{~ms}$, the real-time requirement of the integrated measurement system (delay is not greater than 10ms), shown in Fig 6.

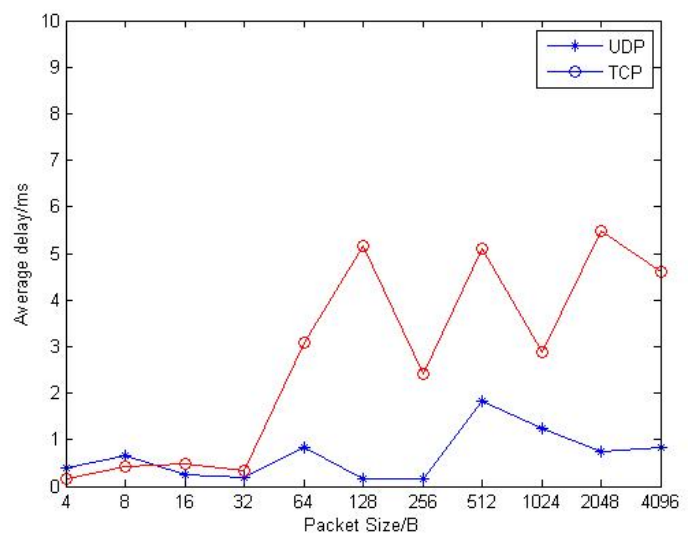

Fig 6 The result of delay between publisher and subscriber 


\section{Summary and future work}

Based on the DDS technology theory, this paper proposes an adaptive QoS spacecraft integrated test system structure. Through various QoS strategies, it can effectively ensure the system's feasibility, real-time and flexibility, so as to effectively solve $\mathrm{C} / \mathrm{S}$ test system problem. It can guarantee the data control and communication transparency between the systems, and can provide an important guarantee for the operation, development and integration of the integrated testing system.

In the future, we intend to implement an integrated DDS technology test system for spacecraft to achieve multiple QoS frames. It improves the real-time and extensibility of the system further more.

\section{References}

[1] Schneider S, Farabaugh B. Is DDS for You? [J]. A Whitepaper by Real-Time Innovations, 2009: p.l-5.

[2] D Esposito C., Russo S., Di Crescenzo D. Performance assessment of OMG compliant data distribution middleware. In proc: IEEE International Symposium on IPDPS, April 2008, p.1-8.

[3] Pardo-Castellote G. Omg data-distribution service: Architectural overview[C] Distributed Computing Systems Workshops, 2003. Proceedings. 23rd International Conference on. IEEE, 2003: p. 200-206.

[4] Object Management Group(OMG). Data Distribution Service for Real. time Systems, version 2.1. Technical Document, August 2007.

[5] Jie Yang. The research of QoS based on IEEE802.16, Master's Degree of Zhejiang University, 2005, p.35 